\title{
ON THE ESTRADA INDEX OF GRAPHS WITH GIVEN NUMBER OF CUT EDGES*
}

\author{
ZHIBIN DU ${ }^{\dagger}$ AND BO ZHOU ${ }^{\ddagger}$
}

Abstract. Let $G$ be a simple graph with eigenvalues $\lambda_{1}, \lambda_{2}, \ldots, \lambda_{n}$. The Estrada index of $G$ is defined as $E E(G)=\sum_{i=1}^{n} e^{\lambda_{i}}$. In this paper, the unique graph with maximum Estrada index is determined among connected graphs with given numbers of vertices and cut edges.

Key words. Estrada index, Cut edge, Spectral moments, Pendant vertex.

AMS subject classifications. 05C50, 05C35, 05C90, 15A18.

1. Introduction. Let $G$ be a simple graph with $n$ vertices. The eigenvalues of the adjacency matrix $\mathbf{A}(G)$ of $G$ are called the eigenvalues of $G$, denoted by $\lambda_{1}, \lambda_{2}, \ldots, \lambda_{n}$. The Estrada index of a graph $G$ is defined as

$$
E E(G)=\sum_{i=1}^{n} e^{\lambda_{i}} .
$$

This concept was proposed in [4], and it found successful applications in biochemistry and in complex networks, see [4-9]. Besides these applications, the Estrada index has also been extensively studied in mathematics, see [2, 3, 10, 11, 13-15]. Among these, Ilić and Stevanović [11] determined the unique tree with minimum Estrada index among the set of trees with given maximum degree. Zhang et al. [13] determined the unique tree with maximum Estrada index among the set of trees with given matching number.

A cut edge of a connected graph is an edge whose removal disconnects the graph. For $0 \leq r \leq n-3$, let $\mathbb{G}(n, r)$ be the set of connected graphs with $n$ vertices and $r$ cut edges, and $G_{n, r}$ the graph obtained by attaching $r$ pendant vertices (vertices of degree one) to a vertex of $K_{n-r}$, where $K_{n}$ is the complete graph on $n$ vertices. Liu et al. [12] characterized the unique graph in $\mathbb{G}(n, r)$ with maximum spectral radius,

${ }^{*}$ Received by the editors on December 14, 2010. Accepted for publication on May 20, 2011. Handling Editor: Bryan Shader. This work was supported by National Natural Science Foundation of China (Grant No. 11071089).

${ }^{\dagger}$ Department of Mathematics, Tongji University, Shanghai 200092, P.R. China (zhibindu@126.com).

${ }^{\ddagger}$ Department of Mathematics, South China Normal University, Guangzhou 510631, P.R. China (zhoubo@scnu.edu.cn). 
which is $G_{n, r}$. In this paper, we determine the unique graph in $\mathbb{G}(n, r)$ with maximum Estrada index, which is also $G_{n, r}$.

2. Preliminaries. Denote by $M_{k}(G)$ the $k$ th spectral moment of graph $G$, i.e., $M_{k}(G)=\sum_{i=1}^{n} \lambda_{i}^{k}$. It is well-known that $M_{k}(G)$ is equal to the number of closed walks of length $k$ in $G$, see [1]. Then

$$
E E(G)=\sum_{i=1}^{n} \sum_{k=0}^{\infty} \frac{\lambda_{i}^{k}}{k !}=\sum_{k=0}^{\infty} \frac{M_{k}(G)}{k !} .
$$

Let $V(G)$ be the vertex set of $G$. Let $M_{k}(G ; u)$ be the number of closed walks of length $k$ starting at $u$ in $G$.

Let $G_{1}$ and $G_{2}$ be two graphs. If $M_{k}\left(G_{1}\right) \leq M_{k}\left(G_{2}\right)$ for all positive integers $k$, then by (2.1), we have $E E\left(G_{1}\right) \leq E E\left(G_{2}\right)$ with equality if and only if $M_{k}\left(G_{1}\right)=$ $M_{k}\left(G_{2}\right)$ for all positive integers $k$. Let $u \in V\left(G_{1}\right)$ and $v \in V\left(G_{2}\right)$. If $M_{k}\left(G_{1} ; u\right) \leq$ $M_{k}\left(G_{2} ; v\right)$ for all positive integers $k$, then we write $\left(G_{1} ; u\right) \preceq\left(G_{2} ; v\right)$. If $\left(G_{1} ; u\right) \preceq$ $\left(G_{2} ; v\right)$ and there is at least one positive integer $k_{0}$ such that $M_{k_{0}}\left(G_{1} ; u\right)<M_{k_{0}}\left(G_{2} ; v\right)$, then we write $\left(G_{1} ; u\right) \prec\left(G_{2} ; v\right)$.

Let $d_{G}(v)$ be the degree of vertex $v$ in the graph $G$.

For a vertex $u$ of a graph $G, G-u$ denotes the graph obtained from $G$ by deleting $u$ and its incident edges. For subset $S$ of the edge set of a graph $G, G-S$ denotes the graph obtained from $G$ by deleting the edges in $S$. For an edge $e$ of the complement of $G, G+e$ denotes the graph obtained from $G$ by adding $e$.

3. Lemmas. Let $H_{1}, H_{2}$ be two non-trivial graphs with $u, v \in V\left(H_{1}\right), w \in$ $V\left(H_{2}\right)$. Let $G_{u}$ be the graph obtained from $H_{1}$ and $H_{2}$ by identifying $u$ with $w$, and $G_{v}$ be the graph obtained from $H_{1}$ and $H_{2}$ by identifying $v$ with $w$.

For positive integer $k$, let $T_{i}(v, k)\left(T_{i}(u, k)\right.$, respectively) be the set of closed walks of length $k$ in $G_{v}\left(G_{u}\right.$, respectively) starting at $v$ (u, respectively) and an edge of $H_{i}$, and ending at an edge of $H_{i}$, where $i=1,2$.

Lemma 3.1. Suppose that $\left(H_{1} ; v\right) \prec\left(H_{1} ; u\right)$. For $i=1,2,\left|T_{i}(v, k)\right| \leq\left|T_{i}(u, k)\right|$ for all positive integers $k$.

Proof. We only prove the case $i=1$. The case $i=2$ is similar.

We may decompose $W \in T_{1}(v, k)$ into two types of closed walks in $G_{v}$ starting at $v$ : (a) a closed walk in $H_{1}$ starting at $v$; (b) a closed walk in $H_{2}$ starting at $v$. Since $\left(H_{1} ; v\right) \prec\left(H_{1} ; u\right)$, we may construct an injection $f_{k}$ mapping a closed walk of length $k$ in $H_{1}$ starting at $v$ into a closed walk of length $k$ in $H_{1}$ starting at $u$. 
Now we construct a mapping $f^{*}$ from $T_{1}(v, k)$ to $T_{1}(u, k)$. Let $W=W_{1} W_{2} \cdots \in$ $T_{1}(v, k)$, where $W_{r}$ for $r \geq 1$ is a closed walk of length $l_{r}$ of type (a) if $r$ is odd, and of type (b) if $r$ is even. Let $f^{*}(W)=f^{*}\left(W_{1}\right) f^{*}\left(W_{2}\right) \cdots$, where $f^{*}\left(W_{r}\right)=f_{l_{r}}\left(W_{r}\right)$ if $W_{r}$ is of type (a), and $f^{*}\left(W_{r}\right)=W_{r}$ if $W_{r}$ is of type (b). Then $f^{*}(W) \in T_{1}(u, k)$. Obviously, $f^{*}$ is an injection from $T_{1}(v, k)$ to $T_{1}(u, k)$. Then the result follows. $\square$

A weak version of the following lemma was given by Zhang et al. [13].

LEMmA 3.2. If $\left(H_{1} ; v\right) \prec\left(H_{1} ; u\right)$, then $E E\left(G_{v}\right)<E E\left(G_{u}\right)$.

Proof. For positive integer $k$, let $S_{1}(k)\left(S_{2}(k)\right.$, respectively) be the set of closed walks of length $k$ in $G_{v}\left(G_{u}\right.$, respectively) containing at least one edge of $H_{1}$ and at least one edge of $H_{2}$. Then

$$
\begin{aligned}
& M_{k}\left(G_{v}\right)=M_{k}\left(H_{1}\right)+M_{k}\left(H_{2}\right)+\left|S_{1}(k)\right|, \\
& M_{k}\left(G_{u}\right)=M_{k}\left(H_{1}\right)+M_{k}\left(H_{2}\right)+\left|S_{2}(k)\right| .
\end{aligned}
$$

We need only to show that $\left|S_{1}(k)\right| \leq\left|S_{2}(k)\right|$ for all positive integers $k$, and it is strict for some positive integer $k_{0}$.

Note that

$$
\left|S_{1}(k)\right|=\left|S_{1}^{(1)}(k)\right|+\left|S_{1}^{(2)}(k)\right|,
$$

where $S_{1}^{(1)}(k)$ is the subset of $S_{1}(k)$ for which every closed walk starts at a vertex in $V\left(H_{1}\right)$, and $S_{1}^{(2)}(k)$ is the subset of $S_{1}(k)$ for which every closed walk starts at a vertex in $V\left(H_{2}\right) \backslash\{w\}$. Similarly,

$$
\left|S_{2}(k)\right|=\left|S_{2}^{(1)}(k)\right|+\left|S_{2}^{(2)}(k)\right|,
$$

where $S_{2}^{(1)}(k)$ is the subset of $S_{2}(k)$ for which every closed walk starts at a vertex in $V\left(H_{1}\right)$, and $S_{2}^{(2)}(k)$ is the subset of $S_{2}(k)$ for which every closed walk starts at a vertex in $V\left(H_{2}\right) \backslash\{w\}$.

Let $W \in S_{1}^{(1)}(k)$ with starting vertex $x$. We may uniquely decompose $W$ into three parts, say $W_{1} W_{2} W_{3}$, where $W_{1}$ is a walk from $x$ to $v$ in $H_{1}, W_{2}$ is a closed walk in $G_{v}$ starting at $v$ and an edge of $H_{2}$, and ending at an edge of $H_{2}$, and $W_{3}$ is a walk from $v$ to $x$ in $H_{1}$. Denote by $k_{r}$ the length of $W_{r}$ for $r=1,2,3$. Then $k_{1}, k_{3} \geq 0$, $k_{2} \geq 2$, and $k_{1}+k_{2}+k_{3}=k$. Let $\mathbf{A}=\mathbf{A}\left(H_{1}\right)$, and let $a_{i j}^{(r)}$ be the $(i, j)$-entry of $\mathbf{A}^{r}$, which is equal to the number of walks of length $r$ from the $i$ th vertex to the $j$ th vertex of $H_{1}$, see [1], where $r \geq 0$. Then

$$
\left|S_{1}^{(1)}(k)\right|=\sum_{\substack{k_{1}+k_{2}+k_{3}=k \\ k_{1}, k_{3} \geq 0, k_{2} \geq 2}} \sum_{x \in V\left(H_{1}\right)} a_{x v}^{\left(k_{1}\right)}\left|T_{2}\left(v, k_{2}\right)\right| a_{v x}^{\left(k_{3}\right)}
$$


Similarly,

$$
\begin{aligned}
& =\sum_{\substack{k_{1}+k_{2}+k_{3}=k \\
k_{1}, k_{3} \geq 0, k_{2} \geq 2}}\left|T_{2}\left(v, k_{2}\right)\right| \sum_{x \in V\left(H_{1}\right)} a_{x v}^{\left(k_{1}\right)} a_{v x}^{\left(k_{3}\right)} \\
& =\sum_{\substack{k_{1}+k_{2}+k_{3}=k \\
k_{1}, k_{3} \geq 0, k_{2} \geq 2}}\left|T_{2}\left(v, k_{2}\right)\right| a_{v v}^{\left(k_{1}+k_{3}\right)} .
\end{aligned}
$$

$$
\left|S_{2}^{(1)}(k)\right|=\sum_{\substack{k_{1}+k_{2}+k_{3}=k \\ k_{1}, k_{3} \geq 0, k_{2} \geq 2}}\left|T_{2}\left(u, k_{2}\right)\right| a_{u u}^{\left(k_{1}+k_{3}\right)} .
$$

By Lemma 3.1, $\left|T_{2}(v, r)\right| \leq\left|T_{2}(u, r)\right|$ for all positive integers $r$. Since $\left(H_{1} ; v\right) \prec$ $\left(H_{1} ; u\right)$, we have $a_{v v}^{(r)} \leq a_{u u}^{(r)}$ for all positive integers $r$, and it is strict for some positive integer $r_{0}$. It follows that $\left|S_{1}^{(1)}(k)\right| \leq\left|S_{2}^{(1)}(k)\right|$, and it is strict for some positive integer $k_{0}$. Similarly, $\left|S_{1}^{(2)}(k)\right| \leq\left|S_{2}^{(2)}(k)\right|$. Therefore $\left|S_{1}(k)\right| \leq\left|S_{2}(k)\right|$ for all positive integers $k$, and it is strict for some positive integer $k_{0}$.

LEMmA 3.3. Let $G_{1}$ and $G_{2}$ be connected graphs with $u \in V\left(G_{1}\right)$ and $v \in V\left(G_{2}\right)$. Let $G$ be the graph obtained by joining $u \in V\left(G_{1}\right)$ with $v \in V\left(G_{2}\right)$ by an edge, and $G^{\prime}$ be the graph obtained by identifying $u \in V\left(G_{1}\right)$ with $v \in V\left(G_{2}\right)$, and attaching a pendant vertex to the common vertex. If $d_{G}(u), d_{G}(v) \geq 2$, then $E E(G)<E E\left(G^{\prime}\right)$.

Proof. Let $H$ be the graph obtained from $G$ by deleting the vertices in $G_{2}$ different from $v$. Let $k \geq 2$ be a positive integer.

For $x \in V(H)$, let $\mathcal{W}_{k}(H ; x)$ be the set of closed walks of length $k$ starting at $x$ in $H$. Then $M_{k}(H ; x)=\left|\mathcal{W}_{k}(H ; x)\right|$. We construct a mapping $f$ from $\mathcal{W}_{k}(H ; v)$ to $\mathcal{W}_{k}(H ; u)$. For $W \in \mathcal{W}_{k}(H ; v)$, we may decompose $W$ into $W=(v u) W^{*}(u v)$, where $W^{*}$ is a closed walk of length $k-2$ starting at $u$ in $H$. Let $f(W)=(u v)(v u) W^{*}$. Obviously, $f(W) \in \mathcal{W}_{k}(H ; u)$ and $f$ is an injection. Since $d_{H}(u)>d_{H}(v)=1$, we have $M_{2}(H ; v)<M_{2}(H ; u)$. Thus, $f$ is an injection but not a surjection for $k=2$. It follows that $(H ; v) \prec(H ; u)$. Since $G\left(G^{\prime}\right.$, respectively) can be obtained from $H$ and $G_{2}$ by identifying $v \in V(H)\left(u \in V(H)\right.$, respectively) with $v \in V\left(G_{2}\right)$, the result follows from Lemma 3.2.

From Eq. (2.1) and noting that $M_{k}(G)$ is equal to the number of closed walks of length $k$ in $G$, we have immediately the following lemma [10].

LEMma 3.4. Let $G$ be a connected graph and e be an edge of its complement. Then $E E(G)<E E(G+e)$.

Let $\mathcal{G}(a, b)$ be the set of graphs obtained by attaching $b$ pendant vertices to some vertices of $K_{a}$, where $a, b \geq 1$. For a graph $G$ with $u, v \in V(G)$, let $r_{k}(G ; u, v)$ be the number of walks of length $k$ from $u$ to $v$ in $G$, and $\mathcal{W}_{k}(G ; u,[v])$ be the set of closed walks of length $k$ starting at $u$ and containing $v$ in $G$. Let $M_{k}(G ; u,[v])=$ $\left|\mathcal{W}_{k}(G ; u,[v])\right|$. 
LEMMA 3.5. Let $G \in \mathcal{G}(a, b)$, where $a \geq 3$ and $b \geq 1$. Let $u$ and $v$ be the two distinct non-pendant vertices in $G$. Suppose that $u$ has $s \geq 1$ pendant neighbors in $G$, and $v$ has no pendant neighbor in $G$. Then $(G ; v) \prec(G ; u)$.

Proof. Let $k$ be a positive integer. Note that

$$
\begin{aligned}
& M_{k}(G ; v)=M_{k}(G-u ; v)+M_{k}(G ; v,[u]), \\
& M_{k}(G ; u)=M_{k}(G-v ; u)+M_{k}(G ; u,[v]) .
\end{aligned}
$$

Since $s \geq 1, G-u$ is a proper subgraph of $G-v$, and thus $(G-u ; v) \prec(G-v ; u)$. We need only to show that $M_{k}(G ; v,[u]) \leq M_{k}(G ; u,[v])$.

For $W \in \mathcal{W}_{k}(G ; v,[u])$, we may decompose $W$ into two parts, say $W_{1} W_{2}$, where $W_{1}$ is the shortest $(v, u)$-section in $W$, and $W_{2}$ is the remaining $(u, v)$-section of $W$. Denote by $w_{1}, w_{2}, \ldots, w_{a-2}$ the common neighbors of $u$ and $v$ in $G$. Let $H$ be the graph obtained from $G$ by deleting the $s$ pendant neighbors of $u$ in $G$. By the choice of $W_{1}$, we know that $W_{1}$ consists of a closed walk starting at $v$ in $H-u$ whose length may be zero and a single edge $v u$, or a walk from $v$ to $w_{i}$ in $H-u$ and a single edge $w_{i} u$ for $1 \leq i \leq a-2$. Note that $r_{k}(G ; u, v)=r_{k}(G ; v, u)[1]$. Then

$$
M_{k}(G ; v,[u])=\sum_{\substack{x \in\left\{v, w_{1}, w_{2}, \ldots, w_{a-2}\right\} \\ k_{1}+k_{2}=k \\ k_{1}, k_{2} \geq 1}} r_{k_{1}-1}(H-u ; v, x) r_{k_{2}}(G ; u, v) .
$$

Similarly,

$$
\begin{aligned}
M_{k}(G ; u,[v]) & =\sum_{\substack{x \in\left\{u, w_{1}, w_{2}, \ldots, w_{a-2}\right\} \\
k_{1}+k_{2}=k \\
k_{1}, k_{2} \geq 1}} r_{k_{1}-1}(G-v ; u, x) r_{k_{2}}(G ; v, u) \\
& \geq \sum_{\substack{x \in\left\{u, w_{1}, w_{2}, \ldots, w_{a-2}\right\} \\
k_{1}+k_{2}=k \\
k_{1}, k_{2} \geq 1}} r_{k_{1}-1}(H-v ; u, x) r_{k_{2}}(G ; u, v) .
\end{aligned}
$$

Note that $H-u \cong H-v$. For positive integer $s, r_{s}(H-u ; v, v)=r_{s}(H-$ $v ; u, u)$, and $r_{s}(H-u ; v, x)=r_{s}(H-v ; u, x)$ if $x \in\left\{w_{1}, w_{2}, \ldots, w_{a-2}\right\}$. Therefore $M_{k}(G ; v,[u]) \leq M_{k}(G ; u,[v])$.

Lemma 3.6. Let $G \in \mathcal{G}(a, b)$, where $a \geq 3$ and $b \geq 2$. If $G \neq G_{a+b, b}$, then $E E(G)<E E\left(G_{a+b, b}\right)$.

Proof. Since $G \neq G_{a+b, b}$, we may choose two non-pendant vertices, say $u$ and $v$, such that both $u$ and $v$ have at least one pendant neighbor in $G$. Suppose that $v$ has $t \geq 1$ pendant neighbors. Let $H$ be the graph obtained from $G$ by deleting the $t$ pendant neighbors of $v$. 
Let $G_{1}$ be the graph obtained from $H$ and the star $S_{t+1}$ on $t+1$ vertices by identifying $u$ with the center of $S_{t+1}$. Note that $G$ can be obtained from $H$ and the star $S_{t+1}$ by identifying $v$ with the center of $S_{t+1}$. By Lemma 3.5, $(H ; v) \prec(H ; u)$. Then $E E(G)<E E\left(G_{1}\right)$ follows from Lemma 3.2. Repeating the transformation from $G$ to $G_{1}$, we may finally have $E E(G)<E E\left(G_{a+b, b}\right)$.

4. Main result. Now we prove our main result.

TheOREM 4.1. Let $G \in \mathbb{G}(n, r)$, where $0 \leq r \leq n-3$. Then $E E(G) \leq E E\left(G_{n, r}\right)$ with equality if and only if $G \cong G_{n, r}$.

Proof. The case $r=0$ follows from Lemma 3.4.

Suppose that $r \geq 1$. Let $G$ be a graph in $\mathbb{G}(n, r)$ with maximum Estrada index. Let $S$ be the set of cut edges in $G$. Obviously, $G-S$ consists of $r+1$ connected components. By Lemma 3.4, all these connected components are complete.

If there exists some edge, say $u_{1} v_{1}$, of $S$ such that $d_{G}\left(u_{1}\right), d_{G}\left(v_{1}\right) \geq 2$, then applying Lemma 3.3 to $G$ by setting $u=u_{1}$ and $v=v_{1}$, we may get a graph in $\mathbb{G}(n, r)$ with a larger Estrada index, a contradiction. Thus, every cut edge of $G$ has exactly one end vertex with degree one, i.e., every cut edge of $G$ is incident to a pendant vertex. Then $G$ is a graph obtained by attaching $r$ pendant vertices to some vertices of $K_{n-r}$, i.e., $G \in \mathcal{G}(n-r, r)$.

If $r=1$, then obviously $G \cong G_{n, 1}$. If $2 \leq r \leq n-3$, then by Lemma 3.6, we have $G \cong G_{n, r} \cdot \square$

\section{REFERENCES}

[1] D. Cvetković, M. Doob, and H. Sachs. Spectra of Graphs - Theory and Application. Academic Press, Inc., New York, 1980.

[2] J.A. de la Peña, I. Gutman, and J. Rada. Estimating the Estrada index. Linear Algebra and its Applications, 427:70-76, 2007.

[3] H. Deng. A proof of a conjecture on the Estrada index. MATCH Communications in Mathematical and in Computer Chemistry, 62:599-606, 2009.

[4] E. Estrada. Characterization of 3D molecular structure. Chemical Physics Letters, 319:713718, 2000.

[5] E. Estrada. Characterization of the folding degree of proteins. Bioinformatics, 18:697-704, 2002.

[6] E. Estrada. Characterization of the amino-acids contribution to the folding degree of proteins. Proteins: Structure, Function and Bioinformatics, 54:727-737, 2004.

[7] E. Estrada and J.A. Rodríguez-Valázquez. Subgraph centrality in complex networks. Physical Review E. Statistical, Nonlinear, and Soft Matter Physics, 71:056103, 9 pages, 2005.

[8] E. Estrada and J.A. Rodríguez-Valázquez. Spectral measures of bipartivity in complex networks. Physical Review E. Statistical, Nonlinear, and Soft Matter Physics, 72:046105, 6 pages, 2005. 
[9] E. Estrada, J.A. Rodríguez-Valázquez, and M. Randić. Atomic branching in molecules. International Jounal of Quantum Chemistry, 106:823-832, 2006.

[10] I. Gutman, E. Estrada, and J.A. Rodríguez-Velázquez. On a graph-spectrum-based structure descriptor. Croatica Chemica Acta, 80:151-154, 2007.

[11] A. Ilić and D. Stevanović. The Estrada index of chemical trees. Journal of Mathematical Chemistry, 47:305-314, 2010.

[12] H. Liu, M. Lu, and F. Tian. On the spectral radius of graphs with cut edges. Linear Algebra and its Applications, 389:139-145, 2004.

[13] J. Zhang, B. Zhou, and J. Li. On Estrada index of trees. Linear Algebra and its Applications, 434:215-223, 2011.

[14] B. Zhou. On Estrada index. MATCH Communications in Mathematical and in Computer Chemistry, 60:485-492, 2008.

[15] B. Zhou and N. Trinajstić. Estrada index of bipartite graphs. International Journal of Chemical Modeling, 1:387-394, 2008. 